\title{
Probabilistic Risk Assessment and Mitigation for UAS Safety and Traffic Management
}

\author{
Jueming $\mathrm{Hu}$
}

\author{
Arizona State University, Tempe, AZ, U.S. \\ Jueming.Hu@asu.edu
}

\begin{abstract}
A probabilistic approach to help future UAS safety and traffic management is proposed. A probabilistic risk-based operational safety bound is first proposed. This will allow UAV to maintain the deviation from the trajectory plan within a "buffer" zone, and keep away from obstacles. Uncertainty quantification, Monte Carlo simulation, and coordinate transformation techniques will be used. Additionally, an algorithm integrating reinforcement learning and the proposed operational safety bound will be developed which is used for collision avoidance and trajectory planning. This will provide a learning ability to UAVs in order to adapt their behavior to changing environments. Also, large-scale UAV management will be studied by using multi-agent generative adversarial imitation learning.
\end{abstract}

\section{Problem Statement}

An unmanned aerial vehicle (UAV) is an aircraft which operates without a human pilot aboard. The Unmanned Aircraft System (UAS) consists of many UAVs, communication links, and controllers. Use of small UAV has been suggested to many areas, such as surveillance, deliveries, search and rescue, traffic monitoring, videography, and precision agriculture. Some operations such as urban air mobility are likely to occur in areas close to buildings or airports.

UAV operation is stochastic in nature and randomness exists in almost every aspect of UTM (Liu \& Goebel, 2018). As a result, trajectory prediction is inexact, because of wind modeling, tracking, navigation, and control errors (Paielli \& Erzberger, 1997). Thus, the inclusion of uncertainty quantification methodology is critical for future safety analysis (e.g., probability of failure). A probabilistic approach for future UAS safety and traffic management is more appropriate in this regard, which is the major motivation of this research.

This research aims to improve both UAS safety and efficiency for the more and more crowded airspace. The starting point is separation assurance between UAVs. From the liter-

Jueming Hu. This is an open-access article distributed under the terms of the Creative Commons Attribution 3.0 United States License, which permits unrestricted use, distribution, and reproduction in any medium, provided the original author and source are credited. ature review, there are two basic types of UAV safety bound: static operational safety bound (i.e., operational safety boundary is predetermined and does not change with UAV operation conditions (Ong \& Kochenderfer, 2016)) and dynamic operational safety bound (i.e., operational safety boundary changes with respect to UAV operation conditions (D'Souza, Ishihara, Nikaido, \& Hasseeb, 2016)). Many important factors, such as UAV performance, positioning and control parameters, and speeds are not explicitly included. The development of such a model is very valuable to evaluate the relationship between operation conditions with operational safety bound and the operational safety bound impact on traffic management. Then the research will focus on applying the proposed separation criterion to trajectory planning in order to enable large-scale low altitude UAS operations.

\section{EXPECTED CONTRIBUTIONS}

The current research will involve developing a dynamic and probabilistic separation criterion for UAS operation. Under the separation criterion, conflict prediction and conflict probability will be studied to evaluate airspace density and improve the efficiency of conflict resolution maneuver. Then, collision avoidance and a framework for multi-UAV imitation learning will be developed.

Contribution 1 - Developing Probabilistic Risk-Based Operational Safety Bound: The determination of the proposed operational safety bound includes measurements of UAV performance, positioning, control characteristics, and onboard flight tracking system. Considering the vehicles heading direction results in an anisotropic vectorized separation boundary shape. Additionally, the proposed operational safety bound is probabilistic and includes various uncertainty sources. For example, errors on the Global Positioning System (GPS), wind magnitude and direction randomness, and UAV maximum deceleration are considered. Due to the anisotropic design and probabilistic risk-based determination, the proposed operational safety bound can balance the safety and efficiency of future UAS operations better than existing static and dynamic operational safety bound concepts.

Contribution 2 - Estimating Conflict Probability under the Proposed Operational Safety Bound: Since the pro- 
posed operational safety bound is anisotropic and dynamic, it's hard to get the analytical solution shown in (Paielli \& Erzberger, 1997) where trajectory uncertainty is approximated as normally distributed and is represented as an ellipse. The proposed method combines Monte Carlo approach and coordination transformation, thus conflict detection changes to a geometric problem of checking overlap of two stadium shape operational safety bounds. An estimation of conflict probability is essential for the evaluation of airspace density, and the determination of the optimal time to initiate a conflict resolution maneuver.

Contribution 3 - UAV Collision Avoidance by Using the Proposed Operational Safety Bound and Reinforcement Learning: UAV collision avoidance can be done by using the proposed operational safety bound to protect itself and keep enough separation away from obstacles. Reinforcement learning is a good approach to do trajectory planning. To integrate reinforcement learning with the proposed operational safety bound, a novel collision cost map is developed. This will provide a learning ability to UAVs in order to adapt their behavior to changing conditions, which is important for fully autonomous UAV flight.

Contribution 4 - Developing a Framework for Multi-UAV Generative Adversarial Imitation Learning: A new framework for multi-UAV generative adversarial imitation learning is developed to enable imitating complex behaviors with multiple cooperative UAVs without much prior knowledge. Provided demonstrations of a set of UAVs interacting with each other within the same environment, the framework is to combine generative adversarial imitation learning and UAV dynamics to distinguish complex cooperative behavior easier and learn the policies from given behavior as much as possible. Moreover, both centralized and decentralized methods are conducted to figure out which is more efficient for multiUAV imitation learning.

\section{RESEARCH PLAN}

The research project is planned to be completed within the scheduled four years starting August 2018. The first year of the study has been completed. In order to efficiently and effectively develop a probabilistic approach for future UAS safety and traffic management, the research plan of the proposed research is presented as follows.

Year One:

- In-depth literature review in UAS traffic management.

- Develop a probabilistic risk-based separation criterion of UAV operation considering prediction, tracking and control errors.

- Compare the developed separation criterion with the existing separation critera.

Year Two:

- Analyze the impact of the proposed UAV operational safety bound on airspace density and traffic control.
- Apply the proposed UAV operational safety bound to collision avoidance.

- Develop an algorithm for UAV trajectory planning by using the proposed operational safety bound and reinforcement learning.

Year Three:

- Integrate multi-agent generative adversarial imitation learning with UAV dynamics and characteristics.

- Develop a framework for multi-UAV generative adversarial imitation learning.

- Evaluate the performance of centralized and decentralized algorithms of multi-UAV generative adversarial imitation learning.

Year Four:

- Do some experiment and test the developed algorithm of multi-UAV generative adversarial imitation learning to ensure safety.

- Optimize the developed algorithm to improve safety and accuracy.

- Write up dissertation.

The progress of each year is expected to be presented in a conference and submitted to a journal for publication within one year.

\subsection{Work Performed}

A novel method to determine probabilistic operational safety bound for rotary-wing UAV traffic management is developed. The key idea is to combine a deterministic model for rotary-wing UAV flying distance estimation to avoid collision and a probabilistic uncertainty quantification methodology to evaluate the risk level (defined as the probability of failure) of separation loss between UAVs.

As for the main concept, the proposed operational safety bound tries to prevent collision for a UAV which is under its normal operation (in Safe Zone in Figure 1). The operational safety bound is determined so that the UAV will not fly into the Failure Zone. The Failure Zone has two possible scenarios: 1) flying into the obstacle directly; 2) flying into the Collision Zone where the UAVs maximum deceleration cannot prevent the vehicle from hitting the obstacle. Between the Safe Zone and Failure Zone, there is a Warning Zone (see Figure 1). The physical meaning of the Warning Zone is that a UAV can have an updated positioning information (and corresponding control action implementation) before reaching the Collision Zone. Thus, corrective actions can be taken by the UAV to avoid the possible collision. In this sense, the Warning Zone can be considered as a buffer zone to provide sufficient time for UAV to mitigate collision risk. Thus, the proposed operational safety bound is defined as the distance between the obstacle and a UAV under normal operation. It includes two parts as shown in Figure 1: Collision Zone and Warning Zone.

As for the probabilistic uncertainty quantification methodology, uncertainty in UAS flight can be classified into two 


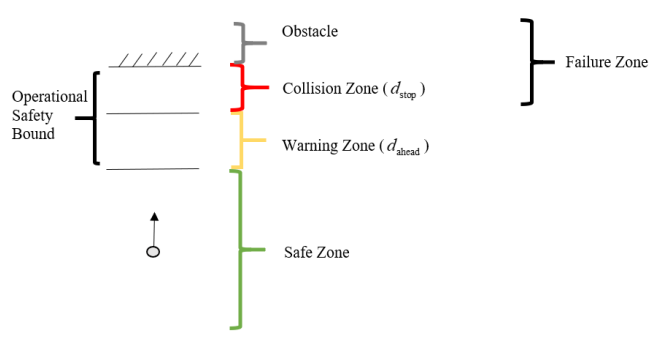

Figure 1. Concept of the proposed operational safety bound.

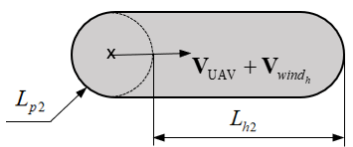

Figure 2. The two-dimensional proposed operational safety bound.

general categories: aleatoric and epistemic uncertainty. Aleatoric uncertainty in the current application refers to the statistical variability of many operational conditions (e.g., UAV speed and wind speed) and system performance (e.g., deceleration of different UAV and positioning system updating frequency). Epistemic uncertainty refers here simply to the measurement error.

Since the parametrized probabilistic model considers UAV's heading direction, it develops an anisotropic operational safety bound. A schematic illustration of the twodimensional proposed operational safety bound is shown in Figure 2. Monte Carlo simulations are used to illustrate the operational safety bound determination with a specified risk level (i.e., probability of failure). Compared to the existing static isotropic (SI) bound and dynamic isotropic (DI) bound, the proposed dynamic anisotropic (DA) bound needs the most information to determine the size including UAV performance, positioning frequency and accuracy, and weather conditions, which means DA bound has the least uncertainty. From Figure 3, it can be seen that uncertainty plays a critical role in determining the operational safety bound size with a certain risk level and knowing additional information for UAVs will greatly reduce the uncertainty. Additionally, the result of conflict probability estimation shows that the airspace based on DA bound has the smallest probability of 9 percent for a conflict to occur while 45 percent for DI bound and 49 percent for SI bound. This indicates that under the same level of risk, the airspace based on DA bound can accommodate more UAVs and is the most efficient.

The proposed probabilistic risk-based operational safety bound for rotary-wing UAV traffic management has been submitted to PHM 2019 and will be presented in September 2019.

\subsection{Remaining Work}

Currently, the research is focused on developing an algorithm of reinforcement learning using the proposed risk-based op-

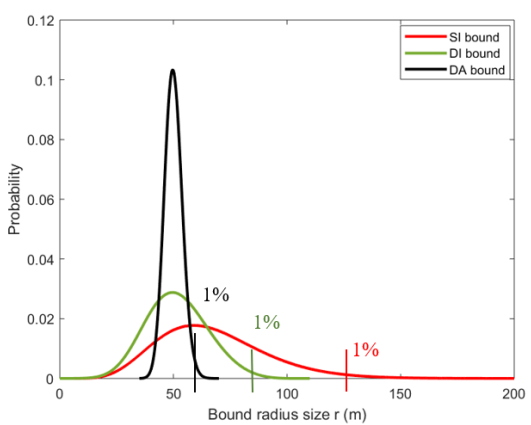

Figure 3. Distribution of operational safety bound size under different methods.

erational safety bound for trajectory planning. It is expected to be completed and be submitted to a journal by March 2020. Then, the research will focus on multi-agent generative adversarial imitation learning and develop the algorithm efficient for multi-UAV. This task will be completed and be submitted to a journal by July 2021. After all the algorithms are developed and simulations are conducted, experiment will be carried out to ensure safety of future UAS operation and airtraffic. The test will be completed by December 2021. The next step is to optimize the developed algorithm from the experiment data and write up dissertation.

\section{Conclusion}

A probabilistic risk-based operational safety bound has been developed, with the contributions of uncertainty quantification and anisotropic geometric design. Then the impact of the proposed bound on the airspace density and traffic control has been studied. In the following three years, trajectory planning and multi-UAV traffic management will be developed. All the proposed work would be beneficial to future UAS sasfety and traffic management.

\section{REFERENCES}

D'Souza, S., Ishihara, A., Nikaido, B., \& Hasseeb, H. (2016). Feasibility of varying geo-fence around an unmanned aircraft operation based on vehicle performance and wind. In 2016 ieee/aiaa 35th digital avionics systems conference (dasc) (pp. 1-10).

Liu, Y., \& Goebel, K. (2018). Information fusion for national airspace system prognostics. In Phm society conference (Vol. 10).

Ong, H. Y., \& Kochenderfer, M. J. (2016). Markov decision process-based distributed conflict resolution for drone air traffic management. Journal of Guidance, Control, and Dynamics, 69-80.

Paielli, R. A., \& Erzberger, H. (1997). Conflict probability estimation for free flight. Journal of Guidance, Control, and Dynamics, 20(3), 588-596. 